\title{
A new model for calculating the critical fluid carrying rate in inclined Wells
}

\author{
Huang QuanHua ${ }^{1}$, Lin XingYu*1 \\ ${ }^{1}$ Petroleum engineering school, Southwest petroleum university, Chengdu, Sichuan, 610500, china
}

\begin{abstract}
In the preparation of gas production plan and the study of gas well proration we often judge the fluid carrying capacity of gas well by the critical fluid carrying rate of the gas well, and then adjust the production plan. At present, spherical droplet model and ellipsoidal droplet model are often used to calculate the fluid flow in gas Wells, but the spherical cap droplet model has not been concerned by relevant scholars. Therefore, we studied the application range of spherical cap droplet model, established relevant mechanical expressions, and finally obtained the formula for calculating the minimum critical liquid carrying rate of spherical cap droplet model. The results show that the spherical cap droplet model cannot be ignored in the conventional droplet model and has a wide range of applications. At the same time, the model is validated by combining with gas Wells of $\mathrm{H}$ gas reservoir, and the model has good applicability.
\end{abstract}

\section{Introduction}

Regardless of the earliest Tuner model or the later Li Min model ${ }^{[1-6]}$, scholars have been studying the fluid carrying capacity of gas Wells in depth, expecting to obtain an accurate and convenient calculation model of critical fluid carrying flow, which can be applied to the design of gas production engineering schemes. The Reynolds number, Ostrol number (gravity/surface tension) and Morton number are often used to characterize droplet morphology. Through a large number of experiments, Grace obtained the droplet shape chart (Figure 1).From the chart, we can

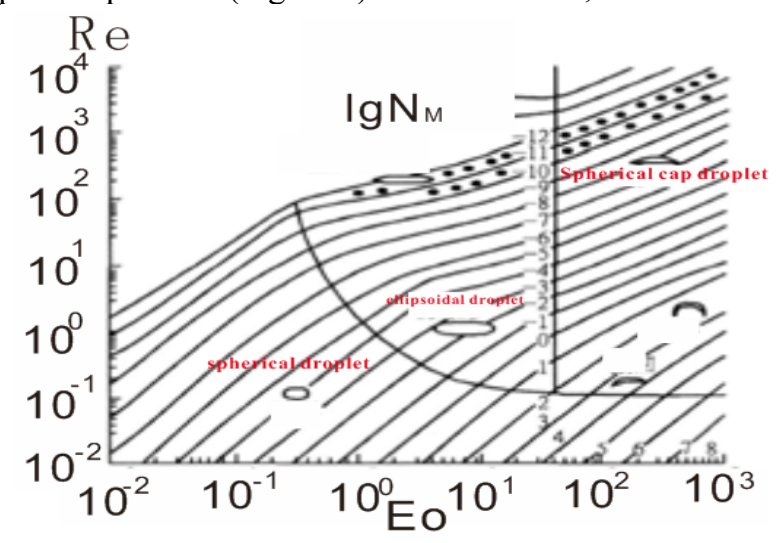

Figure 1. Grace plate clearly see that the spherical cap droplet model is applicable to a wide range, which exists at a low Reynolds number and is almost in the shape of a ball cap at a high Reynolds number. At present, the main calculation models of critical fluid carry flow adopt ellipsoid model. In the previous study, we have derived the critical fluid carry flow model of gas well considering the friction between droplet and borehole wall in detail. But in order to calculate the critical fluid carrying rate of gas well more accurately, we should derive a new method to calculate the critical fluid carrying rate of inclined well.

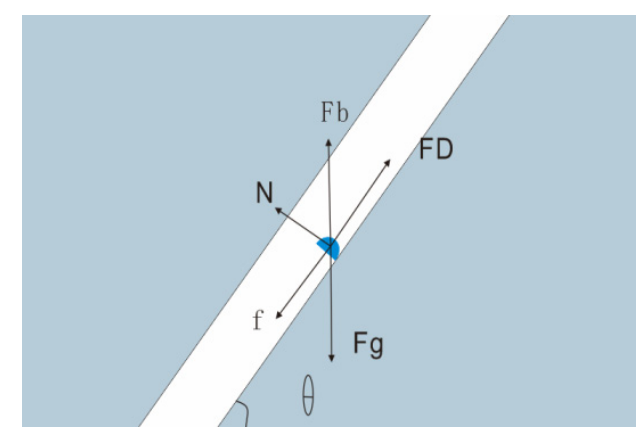

Figure 2 Droplet force diagram

\footnotetext{
*Corresponding author's e-mail: cnlinxingyu@163.com
} 


\section{Formula derivation}

We assume that there is a droplet in the wellbore, regardless of the collision between the droplets; The droplet shape is Spherical cap and the cross section perpendicular to the pipe direction (windward plane) is a circular. Droplet deformation is symmetric along the central axis; Since the liquid phase is mainly water phase, the mass and volume of droplets do not change during migration.

Critical liquid-carrying flow can be understood as the flow required for the gas to fully bring the liquid from the bottom to the surface, usually referring to the largest droplet, while the collision of the droplet with the wellbore should also be considered. The droplet on the wellbore is under the combined action of gravity, buoyancy, drag force, supporting force and friction force, and reaches equilibrium under the critical condition (Fig2).

Five different types of forces applied to the droplet in the wellbore combine to carry it away from the wellbore. When the resultant force just reached the balance, the fluid carrying capacity of gas well began to be insufficient. When the resultant force is insufficient to push the droplet upward along the wellbore direction, the droplet falls back and the gas well accumulates fluid. We can calculate the flow velocity of natural gas in wellbore by analyzing the force on the droplet in equilibrium and connecting the droplet with the natural gas through drag force, and then get the critical fluid carrying rate of the gas well. In the previous study, we have derived the calculation model of the critical carrying capacity of ellipsoid droplet, the difference is that the coefficient of drag is different.

$$
\begin{aligned}
& N+F_{\mathrm{b}} \cos \theta=F_{\mathrm{g}} \cos \theta \\
& F_{\mathrm{D}}+F_{\mathrm{b}} \sin \theta=F_{\mathrm{g}} \sin \theta+f
\end{aligned}
$$

Gravity, buoyancy, drag force and friction force can be expressed as:

$$
\begin{gathered}
F_{\mathrm{g}}=\rho_{\mathrm{l}} \mathrm{g} V=\rho_{\mathrm{l}} \mathrm{g}\left(\frac{\pi}{6} d_{0}^{3}\right) \\
F_{b}=\rho_{\mathrm{g}} \mathrm{g} V=\rho_{\mathrm{g}} \mathrm{g}\left(\frac{\pi}{6} d_{0}^{3}\right) \\
F_{\mathrm{D}}=\frac{1}{2} C_{\mathrm{D}} S \rho_{\mathrm{g}} v_{\mathrm{c}}^{2} \\
f=\lambda N=\lambda\left(F_{\mathrm{g}} \cos \theta-F_{\mathrm{b}} \cos \theta\right)
\end{gathered}
$$

Combine the above expressions and solve:

$$
v_{c}=\left(\frac{2\left(\rho_{l}-\rho_{g}\right) g V(\lambda \cos \theta+\sin \theta)}{C_{D} S \rho_{g}}\right)^{0.5}
$$

In the study of ellipsoid droplet, Wang Yizhong [7] considered the deformed droplet model as conical, and this paper considered the spherical cap property as hemispherical. In contrast, it is more conducive to the calculation of windward surface area. Windward surface area is hemispherical base area:

$$
S=0.3936 \pi d_{0}^{2}
$$

Substituting Equation (8) into Equation (7),

$$
v_{c}=\left(\frac{\left(\rho_{l}-\rho_{g}\right) g d_{0}(\lambda \cos \theta+\sin \theta)}{1.18 C_{D} \rho_{g}}\right)^{0.5}
$$

The above equation refers to the minimum critical fluid carrying velocity solved by the inclined well and spherical cap droplet model. To solve the equation, parameters including gas density, liquid density, droplet diameter, well inclination angle and drag coefficient are required. We can solve the droplet diameter by solving the critical Weber number to solve the maximum diameter of the droplet:

$$
d_{\max }=\frac{W_{e c} \sigma}{\rho_{g} v_{c}^{2}}
$$

where,

$$
v_{c}=\left(W_{e c} \frac{\sigma\left(\rho_{l}-\rho_{g}\right) g(\lambda \cos \theta+\sin \theta)}{1.18 C_{D} \rho_{g}{ }^{2}}\right)^{0.25}
$$

So,

$$
\begin{array}{r}
v_{c}=C_{k, W_{e c}}\left(\frac{\sigma\left(\rho_{l}-\rho_{g}\right)}{\rho_{g}{ }^{2}}\right)^{0.25} \\
C_{k, W_{e c}}=\left(W_{e c} g \frac{(\lambda \cos \theta+\sin \theta)}{1.18 C_{D}}\right)^{0.25}
\end{array}
$$

\section{Parameter determination}

Considering the effect of condensate oil, the density of the liquid phase is not the density of water, but the density of oil-water phase. The weighted average method is adopted to calculate the density of the gas phase:

$$
\rho_{l}=\rho_{\mathrm{o}} \frac{Q_{o}}{Q_{o}+Q_{w}}+\rho_{w} \frac{Q_{w}}{Q_{o}+Q_{w}}
$$

Empirical formula method is often used to determine the gas-liquid surface tension.The gas-liquid surface tension considered in this paper is the gas-liquid surface tension of the three phases of oil vgas and water. First, determine the gas-water surface tension, then determine the oil-gas two-phase tension, and finally determine the gas-liquid two-phase surface tension:

$$
\begin{gathered}
\sigma_{g w}(p, T)=\frac{1.8(410.93-T)}{206}\left(\sigma_{1}-\sigma_{2}\right)+\sigma_{2} \\
\sigma_{1}=76 e^{-0.036257 p} \\
\sigma_{2}=52.5-0.87018 p
\end{gathered}
$$

Where, $\sigma$ is the surface tension, $\mathrm{mN} / \mathrm{m} ; \sigma_{1}$ is Water surface tension at $23.33^{\circ} \mathrm{C}, \mathrm{mN} / \mathrm{m} ; \sigma_{2}$ Water surface tension at $137.78^{\circ} \mathrm{C}, \mathrm{mN} / \mathrm{m} ; T$ is the Temperature, $\mathrm{K} ; \mathrm{P}$ is the Pressure, $\mathrm{MPa}$.

Oil-gas surface tension:

$\sigma_{\text {og }}=[42.4-0.047(1.8 \mathrm{~T}-459.67)-0.267 \mathrm{API}] \exp (-0.10152 \mathrm{p})$

Gas-liquid surface tension:

$$
\sigma=\sigma_{\mathrm{og}} \frac{Q_{o}}{Q_{o}+Q_{w}}+\sigma_{g w} \frac{Q_{w}}{Q_{o}+Q_{w}}
$$


The critical Weber number is calculated by using the general Weber number relation proposed by Wang Zhibin [8] :

$$
W_{e c}=25\left(k^{2}+\frac{1}{k^{4}}-2\right) /\left(7.951-\frac{2.744}{k^{2}}+\frac{0.3077}{k}-5.117 k+0.501 k^{2}\right)
$$

Where,

$$
k=\frac{d_{1}}{d_{0}}
$$

Whrer, ${ }^{d_{1}}$ is the windward diameter, $\mathrm{m}$.

The coefficient of drag is related to the Reynolds number. By comparing the logarithmic deviation squared sum (SSLD), logarithmic deviation square root (RMSLD) and relative error sum (SRE) of different fitting models, Ming Ruiqing [9] believed that the Reza Barati model had higher accuracy when applied to laminar flow. Reza Barati used the polygene genetic code (GP) method to introduce the optimization results of hyperbolic tangent function and calculate the drag coefficient:

$$
\begin{aligned}
& C_{D}=5.4856 \times 10^{9} \tanh \left(\frac{4.3374 \times 10^{-9}}{\operatorname{Re}}\right)+0.0709 \tanh \left(\frac{700.6574}{\mathrm{Re}}\right) \\
& +0.3894 \tanh \left(\frac{74.1539}{\mathrm{Re}}\right)-0.1198 \tanh \left(\frac{7429.0834}{\mathrm{Re}}\right) \\
& +1.7174 \tanh \left(\frac{9.9851}{\operatorname{Re}+2.3348}\right)+0.4744
\end{aligned}
$$

However, for turbulent layer [10], the following method can be used to determine the drag coefficient:

$C_{D}=-3.316 \times 10^{-18} \mathrm{Re}^{3}+7.3 \times 10^{-12} \mathrm{Re}^{2}-4.918 \times 10^{-6} \mathrm{Re}+1.143$

General formula for the calculation of critical liquid carrying flow:

$$
q_{c}=2.5 \times 10^{8} \frac{A P v_{c}}{Z T}
$$

A-Gas well string cross-sectional area, $\mathrm{m}^{2}$.

\section{The example analysis}

We analyzed the liquid carrying capacity of several condensate Wells in $\mathrm{H}$ gas reservoir. Figure 3 shows the daily gas production curve of Well $\mathrm{H} 1$ and the dynamic critical fluid carrying flow calculation curve. The ellipsoidal critical carrying capacity and the Spherical cap critical carrying capacity are calculated respectively. The well went into operation on August 17, 2011, with a slope of $70^{\circ}$.FIG. 4 is a schematic diagram for calculating the dynamic critical fluid carrying rate of Well $\mathrm{H} 2$, which was put into operation on June 3, 2010, with a well inclination Angle of $70^{\circ}$.

The calculation results show that this method can be well applied to the calculation of the critical carrying fluid flow of Well H1. The critical carrying fluid flow calculated by the spherical cap model is less than that calculated by the ellipsoidal model, but the difference is not significant. In the following application process, both of them can predict the critical liquid carrying flow. As can be seen from Figure 3, in the early stage of production of Well H1, normal production was maintained for a long period of time. In August 2016, after gas well fluid carrying capacity was insufficient, the production capacity of Well H1 declined and the production capacity was extremely low in the later stage of production. After the shut-in, the H1 well resumed production. After a short period of production, the gas well production was down again in April 2018, resulting in extremely low production capacity. After shut-in, the well was not opened again for production.

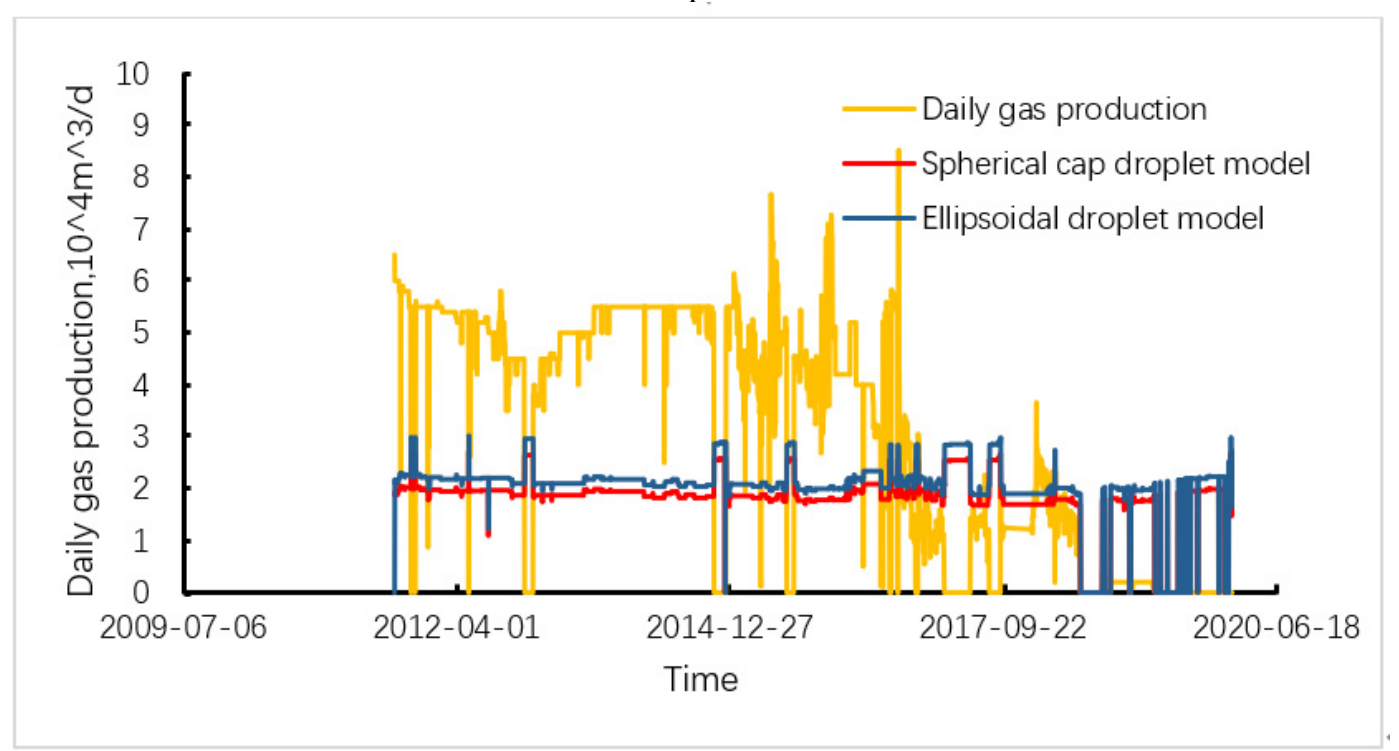

Figure.3 Dynamic diagram for calculation of critical fluid carrying flow in Well H1

The analysis shows that the well was reduced in production due to water invasion. After the water breakthrough to the bottom of the well, the water production increases rapidly, and the water invasion increases the damage to the formation, leading to the decrease of the gas well productivity. After the water breakthrough to the bottom of the well, the gas well starts to fill up with liquid, which aggravates the damage near the well zone and further reduces the gas well productivity until the production is stopped. In other words, water invasion reduces the production capacity of gas well, which leads to the lack of liquid carrying capacity of gas 
well and the production capacity of gas well decreases again.

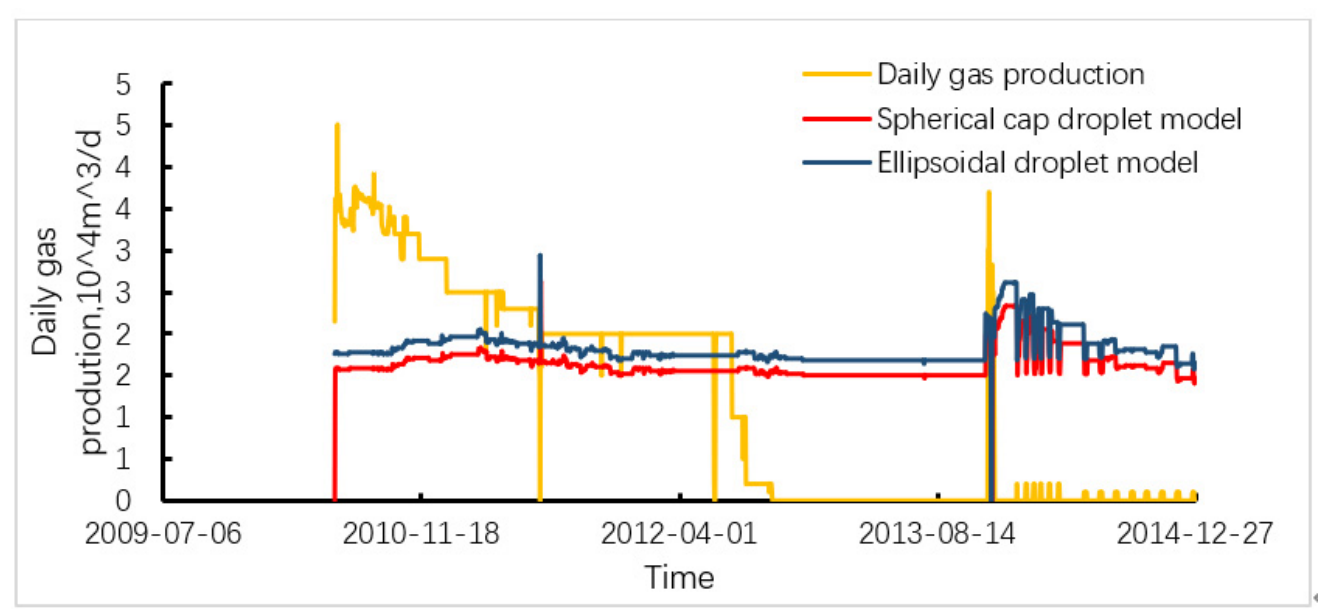

Figure 4 Dynamic diagram for calculating the critical fluid carrying rate of Well H2

It can be seen from Figure 4 that the $\mathrm{H} 2$ well was able to produce normally in the early stage of production, with a certain period of stable production. In July 2012, after the lack of liquid carrying capacity of the well, the production capacity was rapidly reduced, and the gas well was quickly discontinued, and the production capacity was not available after the later production resumed. The well has been producing liquid since it was put into operation, and there is no stable production period. When the liquid carrying capacity of the gas well is insufficient, combined with the influence of the liquid production of the gas well, the liquid accumulation at the bottom of the well is not treated in time, and the gas well output rapidly decreases. In addition, we also analyzed the critical carrying fluid flow of more than 30 inclined Wells. The analysis results show that the ellipsoidal critical carrying fluid flow is slightly larger than the spherical cap critical carrying fluid flow, both of which can accurately predict the critical carrying fluid flow.

\section{Conclusion}

In this paper, a formula for calculating the critical fluid carrying capacity of the ball cap shape is derived, which can be well applied to the study of the fluid carrying capacity of gas Wells and condensate Wells and the preparation of the gas production scheme. Compared with other inclined well models, the friction between droplet and shaft wall is increased in this paper. At the same time, the ball-cap shape model is more consistent with the droplet shape in the actual situation. At the same time, both the model and the ellipsoidal droplet model derived by us can be well applied to the calculation of the critical fluid carrying rate in inclined Wells.

\section{Acknowledgments}

Supported by the National Major Science and Technology Project "Research on percolation Mechanism and Development Technology Policy of Thick Heterogeneous Gas Reservoirs" (No. :2016ZX05027-004-005).

\section{Reference}

1. TURNER R G,HUBBARD M G,DUKLER A E. (1969)Analysis and prediction of minimum flow rate for the continuous removal of liquids from gas wells. Journal of Petroleum Technology, 21(11): 1475-1482.

2. Coleman S B. (1991)A New Look at Predicting Gas Well Load Up. JPT, 329-333.

3. Nosseir M A. (1997) A New Approach for Accurate Prediction of Loading in Gas Well Under Different FlowingConditions.SPE 37408.

4. Li M, Sun L, Li S L, et al. (2001)New view on continuous removal Liquids from Gas Wells. SPE 70016.

5. Li M, Guo P, Zhang M L, et al. (2002) Comparative Study on gas well Continuous Liquid Carrying Model.Journal of Southwest Petroleum College, (04): 30-32+3.

6. Huang Q H, Lin X Y, et al.(2020) Study on critical liquid-carrying flow model in inclined Wells. E3S Web of Conferences, 185:1076.

7. Wang Y Z, Liu Q W.(2007) A New Method for calculating the minimum fluid Critical flow in gas Wells.Daqing Petroleum Geology and Development, $26(6): 82-85$.

8. Wang Z B, Li Y C. (2012) Mechanism of continuous liquid transport in gas Wells. Acta Petroleum Sinica, 33(4): 681-686.

9. Ming R Q. (2019) A general model for predicting the critical fluid carrying rate in condensate Wells. Drilling and Production Technology, 42(6) : 69-72

10. Ming R Q, He H G, Hu Q F. (2008)Calculation Model of critical fluid flow in gas Well under turbulent flow. Geological Science and Technology Information, 37 (3) : 248-252. 